\title{
Preparation of injectable hydroxyapatite/collagen paste using sodium alginate and influence of additives
}

\author{
Taira SATO,,${ }^{* * *}$ Akinori KOCHI, ${ }^{* * * * *}$ Yuki SHIROSAKI, ${ }^{* * *, *}$ Satoshi HAYAKAWA, ${ }^{* * *}$ \\ Mamoru AIZAWA, ${ }^{*}$ Akiyoshi OSAKA** and Masanori KIKUCHI ${ }^{* *}$, \\ ${ }^{*}$ School of Science and Technology, Meiji University, 1-1-1 Higashimita, Tama-ku, Kawasaki 214-8571, Japan \\ ** Biomaterials Unit, National Institute for Materials Science, 1-1 Namiki, Tsukuba, Ibaraki 305-0044, Japan \\ ${ }^{* * *}$ Graduate School of Natural Science and Technology, Okayama University, \\ 3-1-1 Tsushima-naka, Kita-ku, Okayama 700-8530, Japan
}

\begin{abstract}
Preparation of injectable hydroxyapatite/collagen bone-like nanocomposite (HAp/Col) paste utilizing sodium alginate (Na-Alg) and influence of additives on the paste properties were investigated. The HAp/Col at the HAp and collagen mass ratio of 80:20 synthesized by a simultaneous titration method was pelletized to 100-212 $\mu \mathrm{m}$. The HAp/Col powder and that treated with $100 \mathrm{mM} \mathrm{CaCl}_{2}(\mathrm{Ca}-\mathrm{HAp} / \mathrm{Col})$ were used as starting powders. A paste was prepared by mixing of the starting powder and Na-Alg solution at one of several powder/liquid ratios. The paste was directly injected into $100 \mathbf{m M ~ C a C l} \mathbf{~}_{2}$ solution to confirm setting capability, and examined a viscosity, hardening behavior, compressive strength and decay property. In addition, the paste supplemented with an organic acid or a calcium compound as an additive was also prepared to examine influences of the additive on paste properties. The organic acid was chosen from citric, succinic, malic, lactic and glycolic acids, and the calcium compound was chosen from $\mathrm{Ca}(\mathrm{OH})_{2}, \mathrm{Ca}(\mathrm{CO})_{3}, \mathrm{CaSO}_{4} \cdot 0.5 \mathrm{H}_{2} \mathrm{O}$ and calcium citrate.

The prepared paste was set by direct injection to $100 \mathrm{mM} \mathrm{CaCl}_{2}$ solution. The suitable mixing conditions of the paste were a $\mathrm{P} / \mathrm{L}$ ratio of 0.6 at the 90:10 mass ratio of Ca-HAp/Col and solution of low viscous Na-Alg. All additives increased the paste viscosity; however, the mechanisms were different between organic acids and calcium compounds. Organic acids rapidly decreased pH to form Alg gel by deposition of Alg. Contrarily, calcium compounds supplied $\mathrm{Ca}^{2+}$ ions to form egg-box structure for gelation of Alg, and the reaction depended on solubility of compound. Additives also increased decaying time but could not realize anti-decay in the present conditions. The results suggested that the HAp/Col injectable paste can be utilized in rapid prototyping materials and might be good candidate for injectable artificial bone with further improvement in anti-decay property.
\end{abstract}

(C)2013 The Ceramic Society of Japan. All rights reserved.

Key-words: Hydroxyapatite/collagen bone-like nanocomposite, Injectable paste, Artificial bone, Sodium alginate, Anti-decay property, Organic acids, Calcium compounds

[Received May 15, 2013; Accepted July 1, 2013]

\section{Introduction}

Large bone defect, caused by disease or injury, cannot be repaired without using a bone filler. Among bone fillers, autologous bone is still recognized as the best material and used as a gold standard for rapid remodeling including bone regeneration, because autologous bone contains patient's own cells and cytokines as well as hydroxyapatite nanocrystals and collagen with an appropriate nanostructures. Even though, autologous bone transplantation still has considerable problems relating donor site. The healthy donor site is damaged by secondary surgery, and the harvesting amount is limited. The donor site remains without recovery to caused long-term pain and the same site is no longer available for another surgery. Therefore, the development of better artificial bone has been strongly desired.

Artificial bones composed of bioactive ceramics are widely used in medical and dental fields in the forms of dense and porous bodies, ${ }^{1), 2)}$ glanules ${ }^{3)}$ and pastes. ${ }^{4)}$ Among them, pastes have advantages of fluidity to be used in minimally invasive surgery

Corresponding author: M. Kikuchi; E-mail: KIKUCHI. Masanori@nims.go.jp

* Present affiliation is Frontier Research Academy for Young Researchers, Kyushu Institute of Technology. and formability to fit to the bone defect with complicated shape. The pastes currently used clinically are hydroxyapatite (HAp)based; one is utilizing reaction of calcium hydrogen phosphate dihydrtate $\left(\mathrm{CaHPO}_{4} \cdot 2 \mathrm{H}_{2} \mathrm{O}\right)$ and tetracalcium phosphate $\left[\mathrm{Ca}_{4}-\right.$ $\left.\left(\mathrm{PO}_{4}\right)_{2} \mathrm{O}\right]$, and another is utilizing hydration hardening of $\alpha$ tricalicum phosphate $\left[\alpha-\mathrm{Ca}_{3}\left(\mathrm{PO}_{4}\right)_{2}, \alpha\right.$-TCP]. Although the crystallite size of HAp obtained by these reactions is not so large in comparison to that of sintered HAp, they still require very long period to dissolve in our body. A hard HAp block remains patients' body for long time to lead severe problems, such as secondary bone fracture and collapsing. Accordingly, preparation of biodegradable bone paste is expected from surgeons. A biodegradable chelate-setting $\beta$-tricalcium phosphate $(\beta$-TCP) cement using inositol hexaphosphate was prepared and was absorbed in the living body. ${ }^{5)}$ This cement showed good properties but still needs much improvement on biodegradability. Recently, clinical trial data of the hydroxyapatite/collagen nanocomposite (HAp/ $\mathrm{Col})$ having similar chemical composition and nanostructure to bone ${ }^{6)-9)}$ conclude that the $\mathrm{HAp} / \mathrm{Col}$ shows remarkable curative efficacy rate in comparison to $\beta$-TCP artificial bone, Osferion ${ }^{\circledR}$, which is recognized by surgeons as very efficient artificial bone. ${ }^{10)}$ This result suggested that the HAp/Col paste could be much better material than $\beta$-TCP cement. To confer hardening and lubrication properties to the $\mathrm{HAp} / \mathrm{Col}$, sodium alginate $(\mathrm{Na}-\mathrm{Alg}$ ) 
is the one of the best candidates because it has been already investigated as biomaterials for long time by many researchers, ${ }^{11-13)}$ and Na-Alg is hardened by chelating to form egg-box structure with multivalent cations including $\mathrm{Ca}^{2+}$ ion ${ }^{14)}$ which contains in HAp and is known as a good lubricant. ${ }^{15)}$

In the present paper, the HAp/Col paste was prepared by mixing with Na-Alg with and without an additive. Properties of the paste obtained were measured by scanning electron microscopy, compressive strength test, viscosity test, hardening property test and decay property test. The optimal conditions for the HAp/ Col-alginate paste without additive were determined, and influences of the additive on paste properties were investigated.

\section{Materials and methods}

\subsection{Materials}

The HAp/Col with the HAp and collagen mass ratio of 80:20 was prepared by a simultaneous titration method. ${ }^{6), 16)}$ Briefly, for $10 \mathrm{~g}$ of the $\mathrm{HAp} / \mathrm{Col}, 100 \mathrm{~cm}^{3}$ of $400 \mathrm{mM} \mathrm{Ca}(\mathrm{OH})_{2}$ (prepared from alkaline analysis grade $\mathrm{CaCO}_{3}$ (Wako Pure Chemicals Inc., Japan) suspension and $200 \mathrm{~cm}^{3}$ of $120 \mathrm{mM}$ orthophosphoric acid (Reagent grade, Wako Chemicals Inc., Japan) solution with $2.0 \mathrm{~g}$ of type-I porcine dermal collagen (Biomaterial Grade, Nitta Gelatin Inc., Osaka, Japan) solution were simultaneously titrated via tube pumps to the reaction vessel, in which $100 \mathrm{ml}$ of pure water was previously added, with maintaining of temperature at $37^{\circ} \mathrm{C}$ and $\mathrm{pH}$ at 9 . The $\mathrm{HAp} / \mathrm{Col}$ obtained was analyzed by X-ray diffraction (XRD, Rigaku, RINT-Ultima III) and thermogravimetry-differential thermal analysis (TG-DTA, Rigaku, Thermo Plus, Japan). The HAp/Col obtained was compacted at $32 \mathrm{~mm}$ in diameter, $2 \mathrm{~mm}$ in height disk using mold specially designed for squeezing water by uniaxial pressing at $20 \mathrm{MPa}$ and freezedried, the HAp/Col compacted was then crushed into 100-212 $\mu \mathrm{m}$ in size, and collagen molecules in the HAp/Col powder were dehydrothermal cross-linked at 140 degrees for $12 \mathrm{~h}$ under vacuum. Primary hardening reaction expected for the paste was partial dissolution of HAp nanocrystals in the HAp/Col to release $\mathrm{Ca}^{2+}$ ions for gelation of Alg. Thus, presence of $\mathrm{Ca}^{2+}$ ions in liquid phase of the paste was important. However, according to Sotome et al., ${ }^{17)}$ the $\mathrm{HAp} / \mathrm{Col}$ adsorbed $\mathrm{Ca}^{2+}$ and $\mathrm{Mg}^{2+}$ ions in the cell culture medium. To inhibit adsorption of $\mathrm{Ca}^{2+}$ ions in the liquid phase of the paste, the $\mathrm{HAp} / \mathrm{Col}$ powder was stirred in $20 \mathrm{mM} \mathrm{CaCl}_{2}$ solution for 3 days to be saturated for $\mathrm{Ca}^{2+}$ adsorption, followed by filtering, freeze-drying and re-sieving to collect powders $100-212 \mu \mathrm{m}$ in size to obtain Ca-adsorbed HAp/Col (Ca-HAp/Col) powder. The HAp/Col and Ca-HAp/Col powders were observed with scanning electron microscope (SEM, JSM5600LV, JEOL, Japan). Sodium alginates used in the experiment were 80-120 (L-Na-Alg), 300-400 (M-Na-Alg), 500-600 (H-Na-Alg) cP in viscosity at $10 \mathrm{~g} / \mathrm{dm}^{3}$ (Wako Pure Chemicals Inc.) The pastes were prepared by mixing of the Na-Alg solution with $\mathrm{HAp} / \mathrm{Col}$ or $\mathrm{Ca}-\mathrm{HAp} / \mathrm{Col}$ powder under conditions summarized in Table 1. To confirm if the amount of Na-Alg was enough, the paste prepared was directly injected into 100 $\mathrm{mmol} / \mathrm{dm}^{3} \mathrm{CaCl}_{2}$ (Wako Pure Chemicals Inc., Japan) aqueous

Table 1. Concentration of Na-Alg solution at each conditions

\begin{tabular}{crrrrrr}
\hline (HAp/Col)/Na-Alg & \multicolumn{7}{c}{$\mathrm{P} / \mathrm{L}$ ratio } \\
\cline { 2 - 7 } weight ratio & 0.82 & 0.69 & 0.60 & 0.53 & 0.47 & 0.43 \\
\hline $95 / 5$ & 4.8 & 4.0 & 3.5 & 3.0 & 2.7 & 2.4 \\
$90 / 10$ & 9.1 & 7.7 & 6.7 & 5.9 & 5.3 & 4.8 \\
$80 / 20$ & 16.7 & 14.3 & 12.5 & 11.1 & 10.0 & 9.1 \\
\hline
\end{tabular}

solution, this amount is usually enough to form gel by injecting Na-Alg solution, using syringe (Thermo, $2 \mathrm{~mm}$ in caliber). The noodle-like paste was harvested from solution after one minute soaking and observed their conditions by naked eye and fingers.

Gelation of Alg by egg-box formation via $\mathrm{Ca}^{2+}$ would be an essential factor to control setting time and anti-decay property of the paste. Release amounts of $\mathrm{Ca}^{2+}$ can be controlled by an organic acid to dissolve HAp in the HAp/Col or by $\mathrm{Ca}$ compounds with optimal solubility. Therefore, possible candidates for additive would be an organic acid or a Ca compound. An organic acid additive was chosen from citric acid (tricarboxylic compound), succinic acid, malic acid (dicarboxylic compounds with different carbon chain length), lactic acid and glycolic acid (monocarboxylic compounds with different carbon chain length.) Amounts of organic acid were $0.1,1$ and 10 mass \% of HAp/Col according to the preliminary experiment to determine acceptable amount of lactic acid to maintain injectability of $\mathrm{Na}-\mathrm{Alg}$ solution. A Ca compound additive was chosen from $\mathrm{Ca}(\mathrm{CO})_{3}$, calcium citrate (Ca-Cit,) $\mathrm{Ca}(\mathrm{OH})_{2}$ and $\mathrm{CaSO}_{4} \cdot 0.5 \mathrm{H}_{2} \mathrm{O}$ (ordered in small to large solubility.) Amounts of $\mathrm{Ca}$ compound were $0.5 \times, 1.0 \times$ and $2.0 \times$ of equivalent reaction amount $(1.67 \pm 0.07 \mathrm{mmol}$ per $1 \mathrm{~g}$ of $\mathrm{Na}-\mathrm{Alg}$ ) of $\mathrm{Ca}$ ion to Na-Alg preliminary measured. These chemicals for the additive were purchased from Wako Pure Chemicals Inc. The additive except for lactic acid, provided as liquid, was added in a powder form. The Ca-HAp/Col paste, which prepared at the $\mathrm{Ca}-\mathrm{HAp} / \mathrm{Col}$ to the L-Na-Alg mass ratio of 90:10 and the Ca-HAp/Col powder to the Na-Alg solution mass ratio of 0.60 , was used to investigate influences of an additive. One additive chosen from $\mathrm{Ca}$ compounds or an organic acids was added to the $\mathrm{Ca}-\mathrm{HAp} / \mathrm{Col}$ paste while mixing.

\subsection{Viscosity and hardening behavior tests}

Viscosity of the paste prepared was measured according to Ishikawa et al. ${ }^{18)}$ Briefly, $0.1 \mathrm{~cm}^{3}$ of the paste was mixed for $3 \mathrm{~min}$ and a $2 \mathrm{~kg}$ glass plate was placed on the paste in $10 \mathrm{~min}$ after the start of mixing. The spread area was then measured at 10 min after placing the glass.

According to the preliminary test, a hardening behavior test described in the Japanese industrial standard JIS T 6602 for dental zinc phosphate cement could not be applied for the HAp/ $\mathrm{Col}$ and $\mathrm{Ca}-\mathrm{HAp} / \mathrm{Col}$ pastes because of softness of the pastes. Thus, a hardening behavior of the paste was investigated by timedependent viscosity test. The $0.3 \mathrm{~cm}^{3}$ samples, to distinguish a change of value, were packed in silicone tube mold, and were incubated for 1, 2, 3 and 4 days in an incubator maintained its temperature at $37^{\circ} \mathrm{C}$ and relative humidity of $100 \%$. The pastes tested were prepared under conditions summarized in Table 2.

In addition, the influence of additive on initial hardening behavior was measured using $\mathrm{Ca}-\mathrm{HAp} / \mathrm{Col}$ paste with $2.0 \times \mathrm{Ca}-$ $\mathrm{Cit}$ and that with 10 mass \% succinic acid to $\mathrm{Ca}-\mathrm{HAp} / \mathrm{Col}$ powder at 1,6 and $24 \mathrm{~h}$ after incubation by the viscosity test.

Table 2. Powder/liquid ratios for hardening behavior and compressive strength tests

\begin{tabular}{cc}
\hline \multirow{2}{*}{ Molecular weight of Na-Alg } & Mass ratio of HAp/Col to Na-Alg \\
\cline { 2 - 2 } & $90: 10$ \\
\hline Low & $0.60^{*}$ \\
Middle & 0.53 \\
High & 0.53 \\
\hline
\end{tabular}

* for hardening behavior 


\subsection{Compressive strength test}

The samples were prepared according to Table 2. The paste was packed in a silicon tube mold $(5 \mathrm{~mm}$ inside diameter, $6 \mathrm{~mm}$ in height), placed in an incubator for $1,2,3$ and 4 days. The compressive strength of the paste was then measured with a texture analyzer (TA-XT2i, Stable Micro Systems Inc.) with plunger of $10 \mathrm{~mm}$ diameter and head speed of $1 \mathrm{~mm} / \mathrm{min}$.

\subsection{Decay property test}

Decay property for the paste was measured by the procedure in Japanese industrial standard JIS T 0330-4 Bioceramics-Part4: Characterization of calcium phosphate paste. In detail, after $3 \mathrm{~min}$ mixing of raw materials, the paste mixed was packed in the syringe of $4.8 \mathrm{~mm}$ in inner diameter and $16.5 \mathrm{~mm}$ in height. Within 5 min after the start of mixing, the paste was squeezed on wire net with wire diameter of $0.5 \mathrm{~mm}$ and aperture of $2.0 \mathrm{~mm}$, and was soaked into $50 \mathrm{~cm}^{3}$ of $37^{\circ} \mathrm{C}$ phosphate buffered saline (PBS). The paste in PBS was then statically placed at $37^{\circ} \mathrm{C}$ for $72 \mathrm{~h}$ in an incubator. Decay rate was calculated from the final weight of paste left on the net, and decaying time for the paste that completely decayed within $72 \mathrm{~h}$ was also measured.

\section{Results and discussion}

From a result of the TG-DTA measurement, mass ratio of inorganic phases to total mass without adsorbed water for the HAp/Col prepared was $80.2 \%$ and was almost the same as starting material ratio.

Powder X-ray diffraction pattern of the as-prepared HAp/Col showed the low-crystalline HAp, and that of the HAp/Col after heating at $1200^{\circ} \mathrm{C}$ showed presence of 16.7 mass $\% \quad \beta$-TCP; therefore, the inorganic phase of $\mathrm{HAp} / \mathrm{Col}$ obtained was assumed to be a $\mathrm{Ca}$ deficient carbonate containing $\mathrm{HAp}, \mathrm{Ca}_{9.8}\left(\mathrm{CO}_{3}\right)_{0.4^{-}}$ $\left(\mathrm{PO}_{4}\right)_{5.6}(\mathrm{OH})_{2}$.

As shown in Fig. 1, no significant differences of particle morphology between $\mathrm{HAp} / \mathrm{Col}$ and $\mathrm{Ca}-\mathrm{HAp} / \mathrm{Col}$ powders were observed with SEM images as well as specific surface areas of them, $55.1 \pm 3.7 \mathrm{~m}^{2} \cdot \mathrm{g}^{-1}$ for the HAp/Col and $58.2 \pm 3.8 \mathrm{~m}^{2} \cdot \mathrm{g}^{-1}$ for the $\mathrm{Ca}-\mathrm{HAp} / \mathrm{Col}$ powders. Accordingly, adsorption treatment of $\mathrm{Ca}^{2+}$ ion on the HAp/Col did not affect apparent conditions of HAp/Col powder.

The paste poured into $\mathrm{CaCl}_{2}$ aqueous solution hardened immediately as shown in Fig. 2(a), and that collected from the solution had enough strength to handle in surgical and cell culture operation with viscoelasticity as shown in Fig. 2(b); thus, 10 mass \% of Alg in the paste was enough to cross-link via $\mathrm{Ca}^{2+}$ ions. In addition, this property is expected to apply the paste to rapid prototyping system for preparing fine controlled tissue engineering scaffolds.

Figure 3 shows results of viscosity test for various conditions. Viscosity increased (spread area decreased) with increasing in $\mathrm{P} / \mathrm{L}$ ratio and/or increasing in viscosity of Na-Alg used. The most suitable $\mathrm{P} / \mathrm{L}$ ratio of each pastes are determined as Table $\mathbf{3}$, the conditions that spread area revealed $200 \mathrm{~mm}^{2}$, because the paste had good unity and had sufficient but not superabundant water content to allow injection.

Figures $\mathbf{4}$ and $\mathbf{5}$ shows the results of viscosity test for the paste prepared with an organic acid and a Ca compound, respectively. Effect of additive on viscosity depended on its amount. For the organic acid added paste, number of carboxy group(s), size of molecules and/or chelate ability in organic acid had no influences on viscosity; thus, increase of paste viscosity could be only the influence of acid $\mathrm{pH}^{15}$ ) In the case of $\mathrm{Ca}$ compounds addition, the viscosity of the paste increased with increasing in both the amount and solubility of the Ca compounds. These results suggested that viscosity of the paste increased by the cross-link of $\mathrm{Na}-\mathrm{Alg}$ in the paste by dissolved $\mathrm{Ca}^{2+}$ ions from the additive.

At the mass ratio of HAp/Col or Ca-HAp/Col to Na-Alg 95: 5 , the paste prepared was very fragile at 1 day after incubation due to insufficiency of Na-Alg amount to fix the paste. Other NaAlg ratio seemed to be good; however, to maximize the HAp/Col
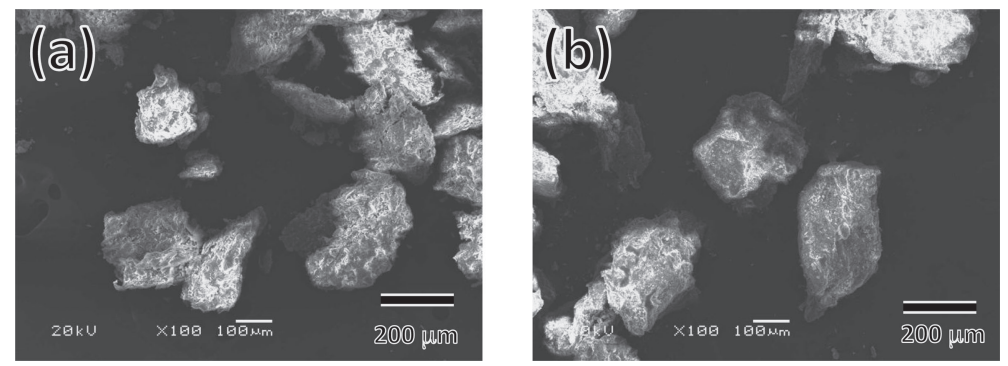

Fig. 1. SEM image of (a) HAp/Col and (b) Ca-HAp/Col.
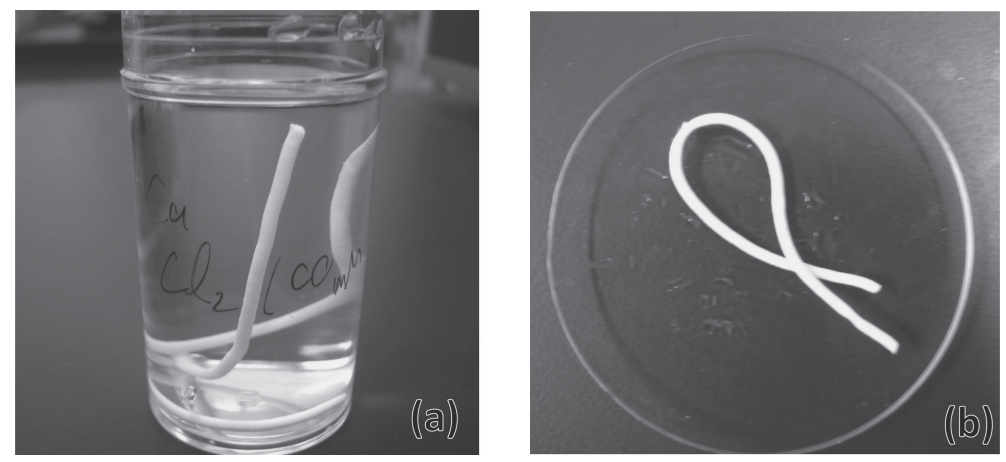

Fig. 2. (a) $\mathrm{Ca}-\mathrm{HAp} / \mathrm{Col}$ paste just after injected into $100 \mathrm{mM} \mathrm{CaCl} 2$ solution. (b) $\mathrm{Ca}-\mathrm{HAp} / \mathrm{Col}$ paste after immersed 100 $100 \mathrm{mM} \mathrm{CaCl} l_{2}$ solution for $1 \mathrm{~min}$. 

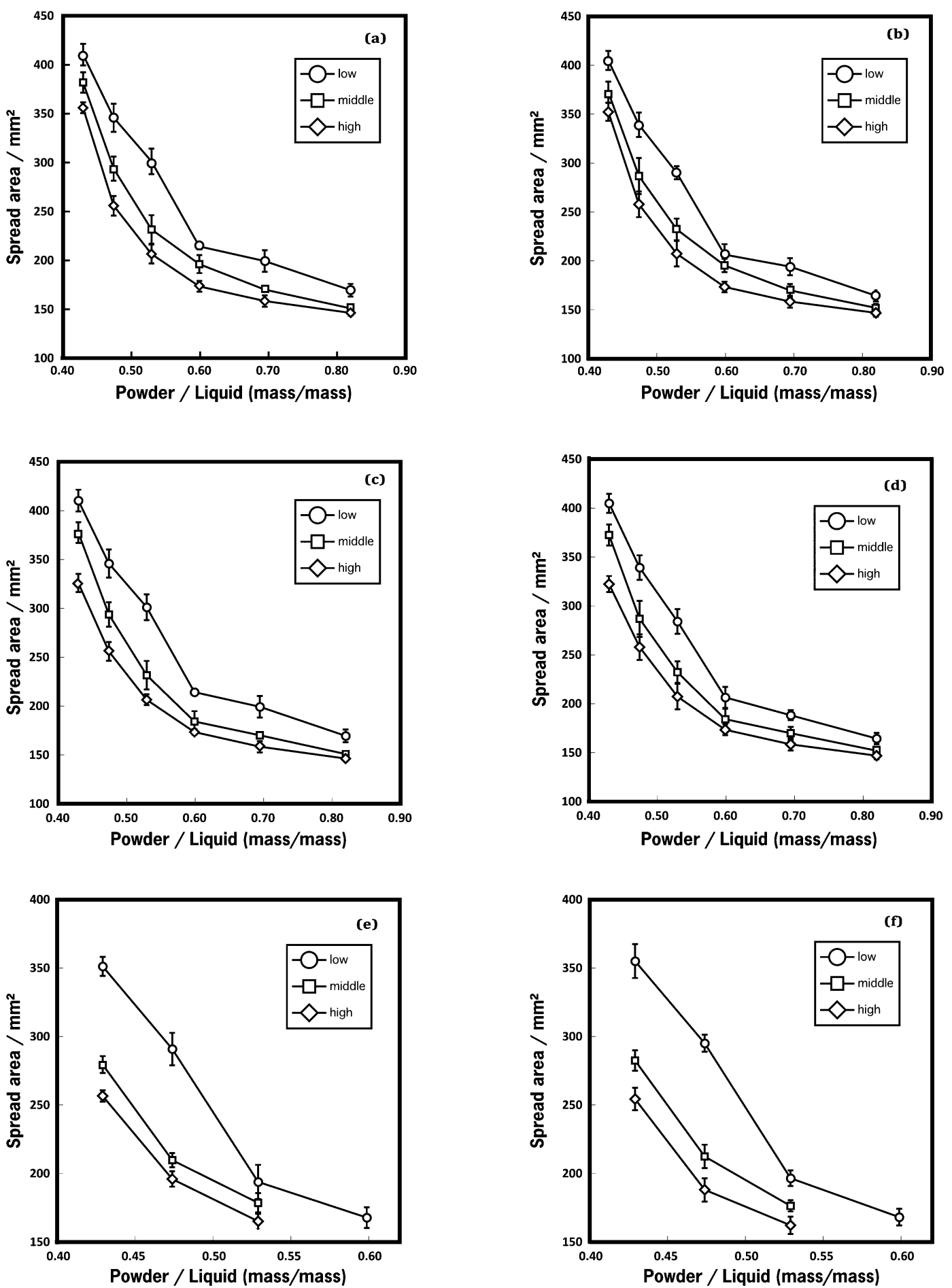

Fig. 3. Spread area, as an index of consistency, of the Hap/Col and Ca-Hap/Col paste prepared by under various conditions. Mass ratio of $\mathrm{Hap} / \mathrm{Col}$ or $\mathrm{Ca}-\mathrm{Hap} / \mathrm{Col}$ to Na-Alg was fixed at 95/5, (a) $\mathrm{Hap} / \mathrm{Col}$ and (b) $\mathrm{Ca}-\mathrm{Hap} / \mathrm{Col}$ paste. Mass ratio of $\mathrm{Hap} / \mathrm{Col}$ or Ca-Hap/Col to Na-Alg was fixed at 90/10, (c) Hap/Col and (d) Ca-Hap/Col paste. Mass ratio of Hap/Col or $\mathrm{Ca}-\mathrm{Hap} / \mathrm{Col}$ to Na-Alg was foxed at 80/20, (e) Hap/Col and (f) Ca-Hap/Col paste.

Table 3. Optimal $\mathrm{P} / \mathrm{L}$ ratio for each conditions

\begin{tabular}{cccc}
\hline \multirow{2}{*}{ Molecular weight of Na-Alg } & \multicolumn{3}{c}{ Mass ratio of HAp/Col to Na-Alg } \\
\cline { 2 - 4 } & $95: 5$ & $90: 10$ & $80: 20$ \\
\hline Low & 0.69 & 0.60 & 0.53 \\
Middle & 0.60 & 0.53 & 0.47 \\
High & 0.60 & 0.53 & 0.47 \\
\hline
\end{tabular}

properties, we concluded that 10 mass $\%$ of Na-Alg would be much better than 20 mass $\%$ of Na-Alg. The time-department changes in viscosity of the $\mathrm{HAp} / \mathrm{Col}$ and $\mathrm{Ca}-\mathrm{HAp} / \mathrm{Col}$ pastes at $\mathrm{Na}-\mathrm{Alg}$ ratio of 10 mass $\%$ are shown in Fig. 6. Viscosities of both pastes increased with time until day 2 ; however, that of the HAp/Col paste reached plateau at day 3 even that of the Ca$\mathrm{HAp} / \mathrm{Col}$ paste was continuously increasing at least till day 4 . 


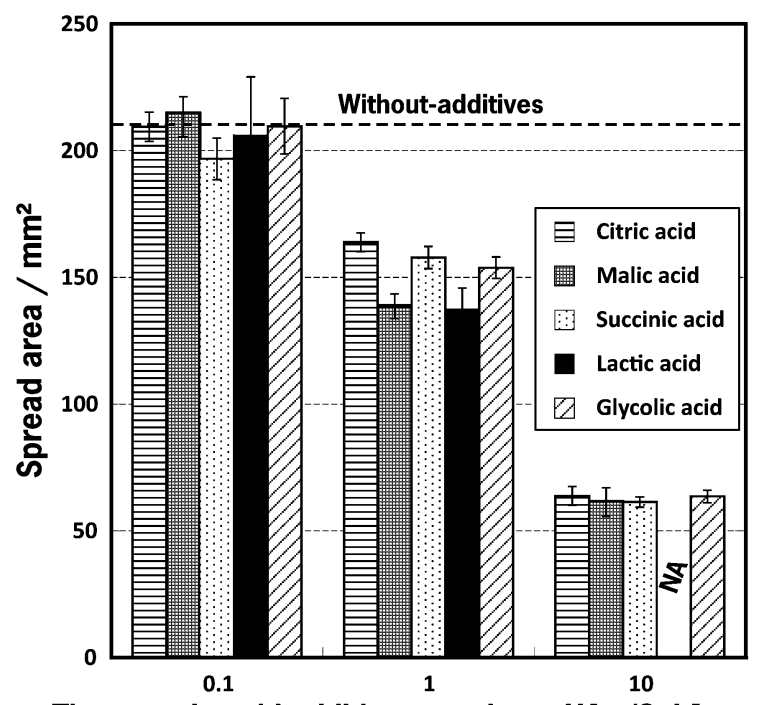

The organic acid addition quantity to $\mathrm{HAp} / \mathrm{Col}$ [mass\%]

Fig. 4. Spread area of organic acid added Ca-HAp/Col paste.

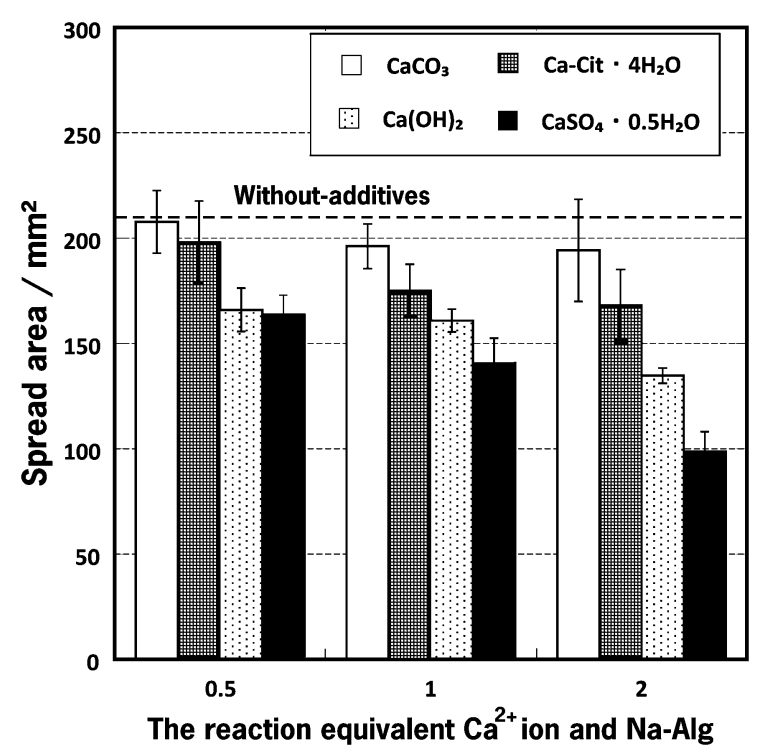

Fig. 5. Spread area of calcium compound added Ca-HAp/Col paste.

These differences could be caused by difference in adsorption behavior of Alg on the HAp/Col and Ca-HAp/Col. Amounts of adsorption site, $\mathrm{Ca}$, on the $\mathrm{Ca}-\mathrm{HAp} / \mathrm{Col}$ particles could be greater than those of $\mathrm{HAp} / \mathrm{Col}$, because of $\mathrm{Ca}^{2+}$ ion adsorption treatment. Initial stage of mixing, large amounts of Alg were adsorbed on the Ca-HAp/Col particles, subsequently amounts of free Alg for gelation decreased from liquid phase of the Ca-HAp/ $\mathrm{Col}$ paste. In the meantime, surface of HAp nanocrystals in both paste started dissolution by chelating effect of Alg. Some of Alg molecules chelating $\mathrm{Ca}^{2+}$ ion(s) started to cross-link via egg-box structures. At this time, lower amounts of free Alg molecules in liquid phase of the $\mathrm{Ca}-\mathrm{HAp} / \mathrm{Col}$ paste allowed formation of smaller amounts of cross-links in comparison to the HAp/Col paste. As a result, viscosity of the Ca-HAp/Col paste was always smaller than that of the HAp/Col paste by day 2. Furthermore, Alg molecules easily cross-linked at neighborhood of the HAp/ Col particles due to existence of large amount of Alg molecules and could inhibit long range Alg gel network formation in the

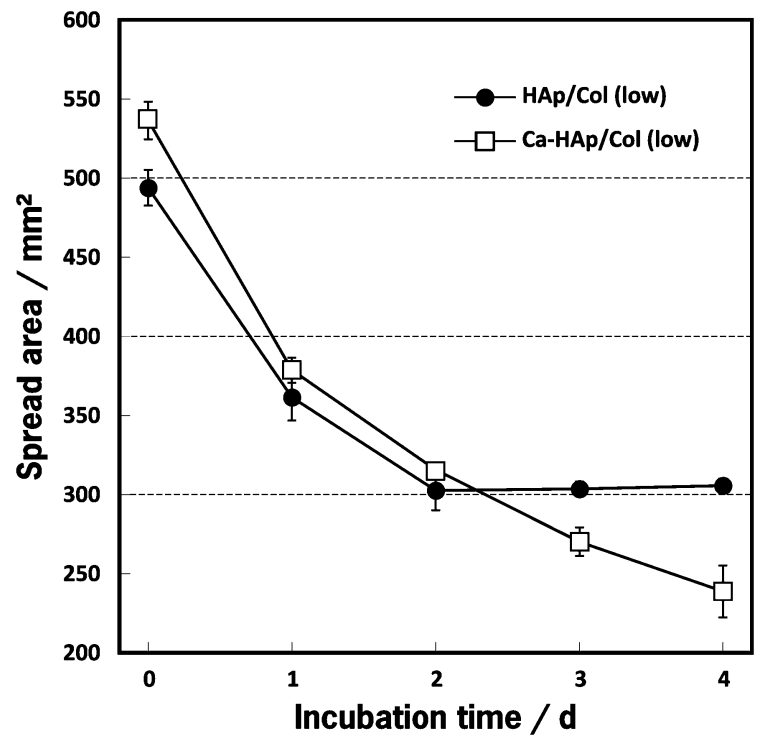

Fig. 6. Spread area of the HAp/col and Ca-HAp/Col pastes prepared at optimum $\mathrm{P} / \mathrm{L}$ ratio as a function of incubation time. Mass ratio of HAp/ Col or Ca-HAp/Col to Na-Alg was set at 90/10.

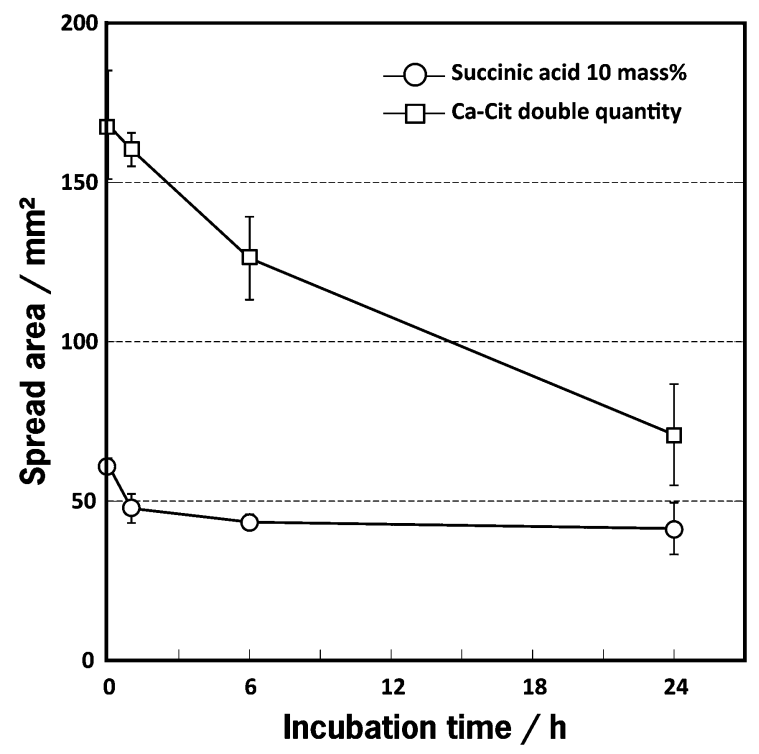

Fig. 7. Hardening behavior of additive-added paste.

$\mathrm{HAp} / \mathrm{Col}$ paste. Contrarily, gradual release of Alg molecules with $\mathrm{Ca}^{2+}$ ions from $\mathrm{Ca}-\mathrm{HAp} / \mathrm{Col}$ particle surfaces could form long-range network initially and form much harder gel in comparison to the $\mathrm{HAp} / \mathrm{Col}$ paste with formation of stronger network at 3 or more days after mixing.

Figure 7 shows the hardening behavior as a function of time for the paste prepared with 10 mass $\%$ succinic acid or $2 \times \mathrm{Ca}-\mathrm{Cit}$. As a trend, viscosity of the pastes increased with time; however, the manner of hardening was very different between the pastes with succinic acid and $\mathrm{Ca}$-Cit. With addition of succinic acid, the paste $\mathrm{pH}$ would decrease rapidly to introduce $\mathrm{Alg}$ gel formation by deposition of Alg from the Alg solution, then the paste viscosity decreased to approximately $60 \mathrm{~mm}^{2}$ in spread are just after mixing and reached plateau, approximately $40 \mathrm{~mm}^{2}$ in spread area in $6 \mathrm{~h}$. Contrarily, viscosity of the paste prepared with $\mathrm{Ca}-\mathrm{Cit}$ increased gently by slow release of $\mathrm{Ca}^{2+}$ ions from $\mathrm{Ca}-$ 


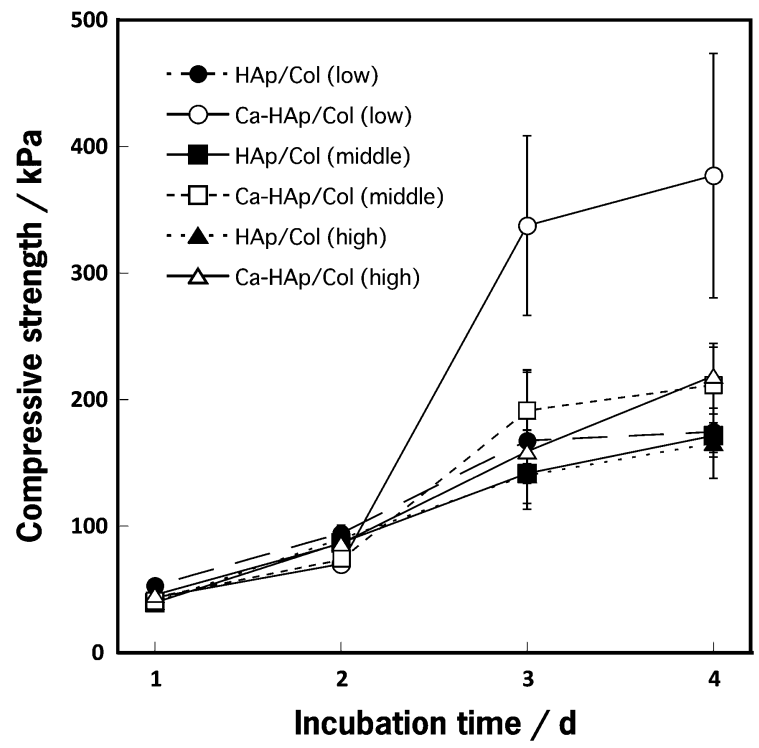

Fig. 8. Changes in compressive strength of the HAp/Col and Ca-HAp/ $\mathrm{Col}$ pastes prepared at optimum $\mathrm{P} / \mathrm{L}$ ratio as a function of incubation time. Mass ratio of HAp/Col or Ca-HAp/Col to Na-Alg was set at 90/10.

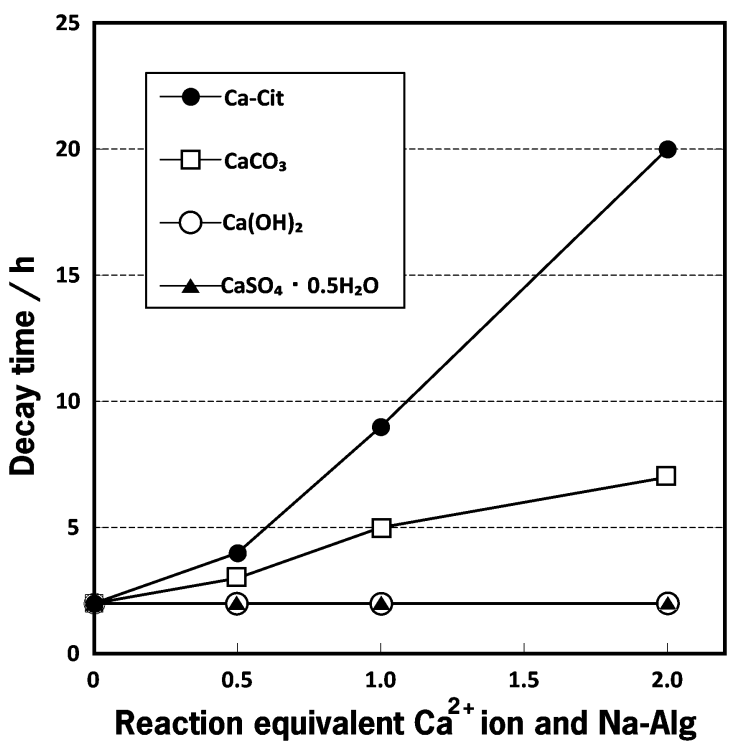

Fig. 9. Decay time of calcium compound added Ca-HAp/Col paste.

Cit; therefore, initial spread area was the same as that of the nonadditive paste. Even though, the paste with $\mathrm{Ca}-\mathrm{Cit}$ decreased its viscosity faster than that of the non-additive paste.

Figure 8 shows the results of compressive strength test. Compressive strengths of all pastes increased with time with similar behavior except for the Ca-HAp/Col paste prepared with L-Na-Alg. The Ca-HAp/Col paste prepared with L-Na-Alg only showed drastic increase of the compressive strength at 3 days after incubation than others. This phenomenon could be caused by differences in dispersion rates of Alg molecules in the pastes due to size of Alg molecules in combination with the usage of $\mathrm{Ca}-\mathrm{HAp} / \mathrm{Col}$ powder as described above.

On the decay property test, all pastes without the additive decayed completely in the D-MEM in less than $2 \mathrm{~h}$, and all pastes with the additive decayed within $24-48 \mathrm{~h}$. Figure 9 shows decay time of the paste with $\mathrm{Ca}$ compound as the additive. Even viscosity of the paste prepared with $\mathrm{CaSO}_{4} \cdot 0.5 \mathrm{H}_{2} \mathrm{O}$ or $\mathrm{Ca}(\mathrm{OH})_{2}$ increased greater than that with other $\mathrm{Ca}$ compounds, no significant differences in decay time were observed between the paste with and without additives. Except for them, decay time showed the same trend to the viscosity change as well as Ca compound solubility. Fundamentally, $\mathrm{Ca}^{2+}$ release from the additives allowed to form Alg network faster than that released from HAp/ $\mathrm{Col}$; thus, viscosity of the paste increased with $\mathrm{Ca}^{2+}$ release rate, solubility. Contrarily, rapid $\mathrm{Ca}^{2+}$ release formed strong Alg gel surrounding of the Ca compound particles, and it inhibited dispersion of $\mathrm{Ca}^{2+}$ ions whole through the paste. Accordingly, Alg coagulates with $\mathrm{Ca}$ compound core formed in the paste to increase viscosity but to decrease unity of the paste.

In the present conditions, the paste composed of HAp/Col and Alg with non-decay property for an injectable artificial bone could not obtained; however, combination of organic acid and $\mathrm{Ca}$ compound would make the injectable artificial bone possible by control both initial viscosity to resist flow to accelerate decay and faster hardening via strong cross-link of Alg molecules via $\mathrm{Ca}^{2+}$ ions.

\section{Conclusion}

The optimal preparation conditions of the HAp/Col paste are that the $\mathrm{P} / \mathrm{L}$ ratio is 0.6 with the $90: 10$ mass ratio of $\mathrm{HAp} / \mathrm{Col}$ powder treated with $\mathrm{CaCl}_{2}$ and low viscous Na-Alg. The paste prepared formed viscoelastic solid by direct injection into $\mathrm{CaCl}_{2}$ aqueous solution. As an additive, organic acid increased paste viscosity very rapidly and $\mathrm{Ca}$ compound accelerate formation of egg-box cross-link in comparison to the non-additive paste. The pastes before hardened were decayed by soaking in D-MEM or PBS, some additive increased time to completely decay.

In conclusion, the $\mathrm{HAp} / \mathrm{Col}$ based paste prepared in the paper would be applied in rapid prototyping with $\mathrm{CaCl}_{2}$ aqueous solution soaking and could be a good candidate for injectable artificial bone with a property to completely incorporate into bone remodeling process after improvement of anti-decay property by using combination of organic acid and $\mathrm{Ca}$ compound.

Acknowledgement This work was supported in part by JST funding for science exchange among Japan, China and Korea.

\section{References}

1) A. Okazaki, T. Koshino, T. Saito and T. Takagi, Biomaterials, 21, 483-487 (2000).

2) U. Ftipamonti, Biomaterials, 17, 31-35 (1996).

3) A. M. Gatti, D. Zaffe and G. P. Poli, Biomaterials, 11, 513-517 (1990).

4) H. Monma and T. Kanazawa, Yogyo Kyokai Shi, 84, 209-213 (1976).

5) S. Takahashi, T. Konishi, M. Nishiyama, M. Mizumoto, M. Honda, Y. Horiguchi, K. Oribe and M. Mamoru, J. Ceram. Soc. Japan, 119, 35-42 (2011).

6) M. Kikuchi, S. Itoh, S. Ichinose, K. Shinomiya and J. Tanaka, Biomaterials, 22, 1705-1711 (2001).

7) S. Itoh, M. Kikuchi, Y. Koyama, K. Takakuda, K. Shinomiya and J. Tanaka, Biomaterials, 23, 3919-3926 (2002).

8) S. Yunoki, T. Ikoma, A. Monkawa, K. Ohta, M. Kikuchi, S. Sotome, K. Shinomiya and J. Tanaka, Mater. Lett., 60, 9991002 (2006).

9) M. Kikuchi, S. Itoh, H. N. Matsumoto, Y. Koyama, K. Takakuda, K. Shinomiya and J. Tanaka, Key Engineering Materials, 240-242, 567-570 (2003).

10) K. Shinomiya, M. Ishizuki, H. Morioka, S. Matasumoto, T. Nakamura, S. Abe and Y. Beppu, Seikei Geka, 63, 921-926 (2012) [in Japanese]. 
11) K. Ishikawa, Y. Miyamoto, M. Kon, M. Nagayama and K. Asaoka, Biomaterials, 16, 527-532 (1995).

12) S. M. Zhang, F. Z. Cui, S. S. Liao, Y. Zhu and L. Han, Jounal of Materials Science; Materials in Medicine, 14, 641-645 (2003).

13) L. Wang, R. M. Shelton, P. R. Cooper, M. Lawson, J. T. Triffitt and J. E. Barralet, Biomaterials, 24, 3475-3481 (2003).

14) E. Stodolak, C. Paluszkiewicz, M. Bogun and M. Blazewicz, J. Mol. Struct., 924-926, 208-213 (2009).

15) K. Y. Lee and D. J. Mooney, Prog. Polym. Sci., 37, 106-126
(2012).

16) M. Kikuchi, T. Ikoma, S. Itoh, H. N. Matsumoto, Y. Koyama, K. Takakuda, K. Shinomiya and J. Tanaka, Compos. Sci. Technol., 64, 819-825 (2004).

17) S. Sotome, T. Uemura, M. Kikuchi, S. Itoh, J. Tanaka, M. Takahashi, T. Tateishi and K. Shinomiya, Key Eng. Mater., 218-220, 153-156 (2002).

18) K. Ishikawa, Y. Miyamoto, M. Takechi, T. Toh, M. Kon, M. Nagayama and K. Asaoka, J. Biomed. Mater. Res., 36, 393399 (1997). 